\title{
Short Term Outcome of Neonates Born With Abnormal Umbilical Cord Arterial Blood Gases
}

\author{
Mousa Ahmadpour-Kacho o,; Yadollah Zahedpasha ${ }^{1}$; Mohsen Hagshenas ${ }^{1}$; Zahra Akbarian \\ Rad $^{1}$; Bahram Sadat Nasseri ${ }^{1}$; Ali Bijani ${ }^{1}$ \\ ${ }^{1}$ Non-Communicable Pediatric Diseases Research Center, Department of Pediatrics, Babol University of Medical Sciences, Babol, IR Iran \\ *Corresponding author: Mousa Ahmadpour-Kacho, Non-Communicable Pediatric Diseases Research Center, Department of Pediatrics, Babol University of Medical Sciences, Babol, \\ IR Iran. E-mail: mousa_ahmadpour@hotmail.com
}

Received: December 16, 2014; Revised: April 9, 2015; Accepted: April 21, 2015

\begin{abstract}
Background: Umbilical arterial blood gas (UABG) analysis is more objective than other methods for predicting neonatal outcome. Acidemic neonates may be at risk for unfavorable outcome after birth, but all neonates with abnormal arterial blood gas (ABG) analysis do not always have poor outcome.

Objectives: This study was carried out to determine the short term outcome of the neonates born with an abnormal ABG.

Patients and Methods: In a cohort prospective study 120 high risk mother-neonate pairs were enrolled and UABG was taken immediately after birth. All neonates with an umbilical cord $\mathrm{pH}$ less than 7.2 were considered as case group and more than 7.2 as controls. Outcomes like need to resuscitation, admission to newborn services and/or NICU), seizure occurrence, hypoxic ischemic encephalopathy (HIE), delayed initiation of oral feeding and length of hospital stay were recorded and compared between the two groups. P value less than 0.05 was considered as being significant.

Results: Comparison of short term outcomes between normal and abnormal ABG groups were as the fallowing: need for advanced resuscitation 4 vs. $0(\mathrm{P}=0.001)$, NICU admission 16 vs. $4(\mathrm{P}=0.001)$, convulsion 2 vs. 0 ( $\mathrm{P}=0.496)$, HIE 17 vs. 4 ( $\mathrm{P}=0.002)$, delay to start oral feeding 16 vs. $4(\mathrm{P}=0.001)$, mean hospital stay 4 vs. 3 days $(\mathrm{P}=0.001)$. None of the neonates died in study groups.

Conclusions: An umbilical cord PH less than 7.2 immediately after birth can be used as a prognostic factor for unfavorable short term outcome in newborns.
\end{abstract}

Keywords: Umbilical Arterial Blood Gas; pH; Propionic Acidemia; Assessment, Patient Outcome

\section{Backgrounds}

Various clinical and laboratory variables have been used to predict the development of poor neonatal outcome immediately after birth.

$\mathrm{pH}$ assessment of cord ABG analysis is an objective diagnostic method for high risk newborns that may be at risk for poor neurologic outcome like birth asphyxia (1). Apgar score is not an objective method to assess the outcome. Most cerebral palsy patients had normal Apgar score at birth (2).

It has since become widely accepted that umbilical cord blood gas analysis can provide important information about the past, present and possibly the future condition of the infant. Umbilical cord blood gas analysis is now recommended in all high risk deliveries by both the British and American Colleges of Obstetrics and Gynecology $(3,4)$, and in some centers it is practiced routinely following all deliveries.

Low cord $\mathrm{pH}$ in infants who are vigorous at birth and free of cardiopulmonary compromise does not indicate an increased risk of adverse outcome (5). A good blood gas in a depressed newborn should alert the neonatologist to search more diligently for other causes of neonatal depression like sepsis, trauma or congenital abnormalities (6).

Considerable effort has been spent to determine risk factors and the role of intrapartum asphyxia for adverse neonatal outcome (7). Based on different studies, mean values of umbilical cord blood $\mathrm{pH}$ range from 7.25-7.28 (8). A baby with abnormal umbilical cord $\mathrm{pH}$ does not always present with poor outcome.

\section{Objectives}

So this study was carried out to determine the short term outcome after birth in the neonates born with an abnormal umbilical cord $\mathrm{pH}$.

\section{Patients and Methods}

This study is a prospective observational cohort study carried out at Rohani hospital, affiliated to Babol Univer-

Copyright (C) 2015, Growth \& Development Research Center. This is an open-access article distributed under the terms of the Creative Commons Attribution-NonCommercial 4.0 International License (http://creativecommons.org/licenses/by-nc/4.0/) which permits copy and redistribute the material just in noncommercial usages, provided the original work is properly cited. 
sity of Medical Sciences in Babol, Mazandaran, Iran. The study was approved by the university ethic committee and informed consents were taken from the parents. All term neonates born between November 2011 and June 2012 by Cesarean Section (C/S) to a high risk mother based on neonatal resuscitation program (NRP) (9) entered the study; an umbilical cord arterial blood sample was taken according to the AAP protocol. All samples were sent in pre-heparinized syringes and prepared under aseptic condition and occupational safety. The samples were analyzed by ABG analyzer (Gem Premier, USA) 30 minutes after birth. Neonates with umbilical cord $\mathrm{pH}$ less than 7.2 were considered as cases and neonates with umbilical cord $\mathrm{pH}$ more than 7.2 as control group.

Data including $\mathrm{pH}, \mathrm{PO}^{2}, \mathrm{PCO}^{2}, \mathrm{BE}$ and bicarbonate along with demographic data like maternal risk factor and age, gestational age, weight and sex of neonates were recorded by a questioner.

Inclusion criteria were term delivery to a high risk mother based on neonatal resuscitation program (NRP) schedule. Extraction criteria were prenatal infection and any major congenital anomaly. Considering $d=20$ with an $80 \%$ power and $95 \%$ confidence interval, sample size was calculated 120 (60 mother-neonate pairs in each arm).

\subsection{Outcome Variables}

\subsubsection{Need to Resuscitation}

On the basis of NRP, we began to resuscitate neonates with meconium stained amniotic fluid, lack of spontaneous or effective respiratory effort and lack of appropriate muscular tone.

\subsubsection{Need to Being Admitted to Neonatal Ward or NICU}

Neonates who required positive pressure ventilation were admitted to newborn services and/or NICU.

\subsubsection{Convulsion}

Diagnosis of convulsion made by clinical observation by attending physician or nurse's reports. All neonates with convulsion were admitted and treated in neonatal ward or NICU.

\subsubsection{Encephalopathy}

Any asphyxiated neonate with neurologic signs and symptoms defined and classified based on the Sarnat and Sarnat staging system (10).

\subsubsection{Delayed Start of Oral Feeding and/or Feeding In- tolerance}

Because of feeding intolerance or respiratory disorder, oral feeding started after stabilization. All neonates admitted to the NICU and/or SCN were kept NPO and IV fluid was started.

\subsubsection{Hospital Stay}

This period is calculated from birth to discharge and declared as days.

\subsubsection{Death}

Any cases of death in both groups were recorded.

\subsection{Statistical Analysis}

Data are analyzed by SPSS, version 18 ; $\mathrm{X}^{2}$ test, MannWhitney and Spearman test were used. P less than 0.05 was considered as being significant.

\section{Results}

The baseline demographic data of the two groups are shown in Table 1. These variables did not show any significant differences between the two groups. Comparison of ABG parameters in two neonatal groups with normal and abnormal umbilical cord $\mathrm{pH}$ are as in Table 2. According to this table, differences between $\mathrm{pH}, \mathrm{PCO}^{2}, \mathrm{PO}^{2}, \mathrm{BE}$ and bicarbonate were significant.

Comparison of short term outcomes including need to resuscitation, convulsion, encephalopathy and its severity, neonatal ward and NICU admission, delay in commencement of feeding and length of hospital stay clearly were better in non-acidemic neonates than in acidemic newborns (Table 3). Need to resuscitation including initial steps in acidemic neonates was higher than the non-acidemic neonates ( 42 vs. 13 neonates; $\mathrm{P}=0.001$ ) but there was no significant difference for PPV requirement (advanced resuscitation) between acidemic and non-acidemic neonates ( 4 vs. 0 neonates; $\mathrm{P}<0.119$ ).

One neonate needed chest compression, had cord arterial pH 6.99 and BD 12.7. This case stayed in NICU for 11 days. We had two cases of convulsion. First case had horizontal deviation of eyes along with apnea. Second case had tonic lower limb extension like decerebrated posture along with bradycardia. This case had paroxysmal tachycardia with hypertension. He received midazolam to control the seizure. Although in acidemic neonates we have 3.3\% convulsive disorders but in non-acidemic hewborns there were no cases of seizure. So in comparison with non-acidemic neonates there was no significant difference in seizure between the two groups $(P=0.496)$.

\subsection{Hypoxic-Ischemic Encephalopathy}

There were 21 cases of encephalopathy in this study. Signs of HIE often had been presented during first hours or days after birth. Some patients presented with mild signs such as decreased alertness, irregular respiratory efforts, and hypotonicity along with normal pupillary reflex.

Convulsive disorders as signs of moderate encephalopathy had been presented in two cases of our patients who had perinatal acidemia and in 24 to 48 hours after birth 
their neurologic symptoms became remarkable.

In acidemic group 17 (28.3\%) cases had encephalopathy but in nonacidemic group only 4 (6.7\%) cases were encephalopathic with a calculated risk ratio of 4.25 . So there was significant relationship between encephalopathy and perinatal acidemia $(\mathrm{P}=0.002)$.

Severity of encephalopathy: Fifteen cases of grade 1 HIE occurred in acidemic group versus only 4 cases in non-acidemic group. On the other hand, in acidemic group we had two cases of grade 2 HIE but in non-acidemic group there were no cases of severe HIE. Also we found a linear relationship between the intensity of acidemia and severity of encephalopathy and all results of parametric tests were like nonparametric results $(\mathrm{P}=0.002)$.

\subsection{Death}

There was no case of death in the two groups of the study.

\subsection{Time of Oral Feeding Initiation}

In acidemic group approximately half of the patients had oral feeding intolerance in the first hour after birth, 14 cases after two hours and 5 cases after four hours could tolerate oral feeding. These patients received D/W 10\% IV and were transferred to the ward. Commencement and Tolerance of Oral Feeding in Patients With Abnormal Umbilical Cord $\mathrm{pH}$ has been shown in Figure 1.

\subsection{Delay in Oral Feeding Initiation}

In acidemic group we commenced oral feeding for 6 cases at 24 hours of life and for 3 cases at 48 hours of life. One case had oral feeding intolerance until 5 days after birth. Finally 31 (51.7\%) cases had oral feeding and 29 (48.3) cases had oral feeding intolerance in the first hour of life. In non-acidemic group 4 neonates had begun feeding at 2, and 2 cases after 4 hours of life, and 3 neonates gradually begun breast feeding after 48 hours and in one case at 96 hours of life. Finally in 50 (83.3\%) cases of newborns in non-acidemic group oral feeding had begun in the first hour of life (Figures 2 and 3). Also Duration of NICU and Neonatal Ward Stay in Non-Acidemic Neonates has been shown in Figure 4.

\subsection{Hospital Stay}

Mean hospital stay in acidemic neonates was 3.78 \pm 3.07 days. In acidemic neonates the length of hospital stay had reverse relationship with umbilical cord $\mathrm{pH}(\mathrm{r}=-0.363, \mathrm{P}$ $<0.001), \mathrm{NaHCO}_{3}(\mathrm{r}=-0.304, \mathrm{P}<0.001)$ and $\mathrm{BE}(\mathrm{r}=-0.456$, $\mathrm{P}<0.001)$ whereas there was no linear relationship with $\mathrm{PO}^{2}$.The result of nonparametric tests was the same as the parametric tests (Figures 3 and 4).

\subsection{Risk Factors}

Meconium stained amniotic fluid was the most common risk factor which has been seen in both groups. Premature rapture of membranes (PROM) was the second most common risk factor in our study.

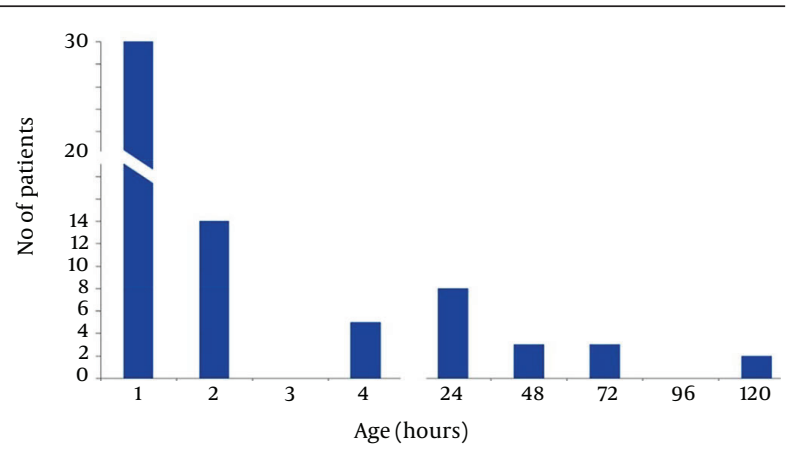

Figure 1. Commencement and Tolerance of Oral Feeding in Patients With Abnormal Umbilical Cord pH

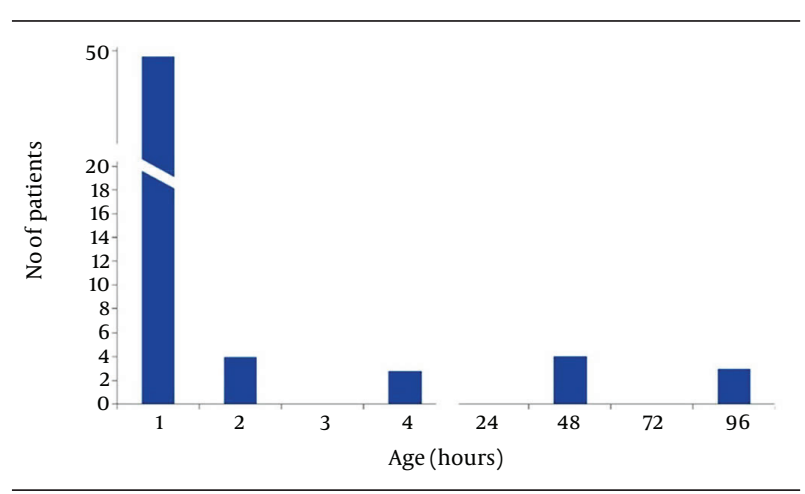

Figure 2. Commencement and Tolerance of Oral Feeding in Neonates With Normal Umbilical Cord $\mathrm{pH}$

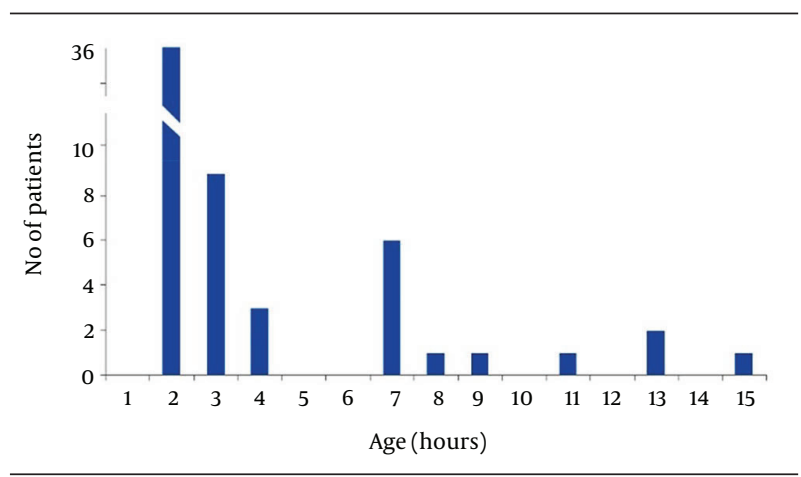

Figure 3. Duration of NICU and Neonatal Ward Stay in Acidemic Neonates

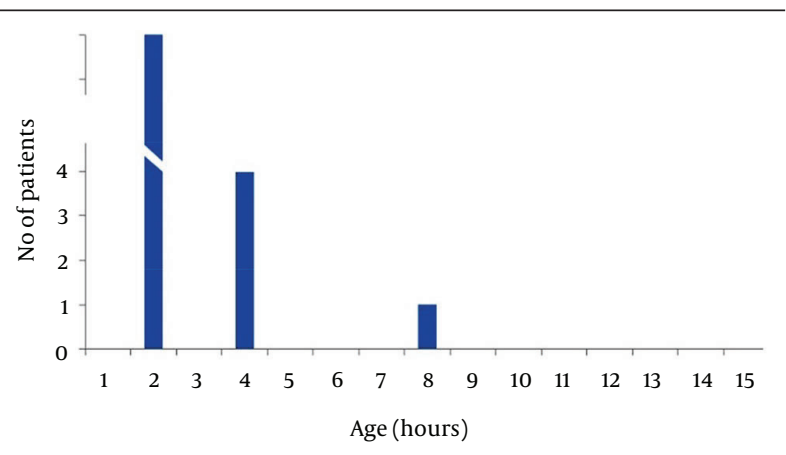

Figure 4. Duration of NICU and Neonatal Ward Stay in Non-Acidemic Neonates 
Ahmadpour-Kacho M et al.

\begin{tabular}{|c|c|c|c|}
\hline Variables & Abnormal pH Group ${ }^{a}$ & Normal pH Group $^{a}$ & Pvalue \\
\hline Gender $^{b}$ & & & 0.144 \\
\hline Female & $34(56.6)$ & $25(41.7)$ & \\
\hline Male & $26(43.3)$ & $35(58.3)$ & \\
\hline Weight $^{\mathrm{c}}, \mathrm{gr}$ & $32640 \pm 554$ & $3204 \pm 370$ & 0.622 \\
\hline Gestational age ${ }^{\mathrm{C}}$, week & $39 \pm 1$ & $38 \pm 1$ & 0.549 \\
\hline Maternal age ${ }^{c}$, year & $28.8 \pm 2$ & $25 \pm 4.2$ & 0.001 \\
\hline
\end{tabular}

\begin{tabular}{|c|c|c|c|}
\hline Parameters & Abnormal pH Group ${ }^{b}$ & Normal pH Group $^{b}$ & Pvalue \\
\hline pH & $7.135 \pm 0.066$ & $7.284 \pm 0.037$ & $>0.001$ \\
\hline $\mathbf{P O}^{2}$, Torr & $11 \pm 6$ & $14 \pm 6$ & 0.003 \\
\hline PCO $^{2}$, Torr & $63.7 \pm 13.7$ & $52.1 \pm 7.9$ & 0.001 \\
\hline BE, mmol & $-8.34 \pm 3.57$ & $-2.48 \pm 2.34$ & 0.001 \\
\hline Bicarbonate, mEq & $21.36 \pm 3.54$ & $24.13 \pm 3.25$ & 0.001 \\
\hline
\end{tabular}

a Data are presented for $\mathrm{n}=60$.
$\mathrm{b}$ Data are presented as Mean $\pm S D$

\begin{tabular}{|c|c|c|c|}
\hline Outcome Variables & Abnormal pH Group ${ }^{\text {a }}$ & Normal pH Group ${ }^{a}$ & P value \\
\hline Need for resuscitation & $42(70)$ & $13(21.7)$ & $<0.001$ \\
\hline Need for PPV & $4(6.7)$ & $0(0)$ & 0.119 \\
\hline Convulsion & $2(3.3)$ & $0(0)$ & 0.496 \\
\hline HIE & $17(28.3)$ & $4(6.7)$ & 0.002 \\
\hline Need for NICU admission & $16(26.7)$ & $3(5)$ & 0.001 \\
\hline Delays in oral feeding initiation unti an hour & $29(48.3)$ & $10(17.7)$ & 0.012 \\
\hline Hospital stay & $3.78 \pm 3.07^{b}$ & $2.28 \pm 0.98^{b}$ & $<0.001$ \\
\hline
\end{tabular}

a Data are presented for $\mathrm{n}=3$ and as No. (\%).

$\mathrm{b}$ Data are presented as Mean \pm SD.

\section{Discussion}

Our study which has been carried out for comparison of short outcomes in normal and abnormal umbilical cord $\mathrm{pH}$ groups, showed that short outcomes including need to resuscitation, encephalopathy, convulsion, NICU and neonatal ward admission and length of hospital stay, delay in commencement of oral feeding in abnormal umbilical cord $\mathrm{pH}$ group are more common than in normal umbilical cord $\mathrm{pH}$ group (11).

Andres et al. assessed ninety three neonates for the correlation between the umbilical cord $\mathrm{PCO}^{2}, \mathrm{PO}^{2}, \mathrm{pH}$, bicarbonate and $\mathrm{BD}$ with death, need to cardiopulmonary resuscitation, convulsion, RDS, PDA, NEC, IUGR, sepsis and HIE. Although in their study there was no correlation between sepsis, RDS, NEC, PDA and UABG parameters, need to cardiopulmonary resuscitation, need to intubation, convulsion, HIE and IUGR were correlated with low UABG pH (11). In their study $\mathrm{PO}^{2}$ had no correlation with outcomes. In our study, abnormal UABG pH had no significant correlation with convulsion.

Goldaber and colleagues studied the association between umbilical arterial acidosis and neurologic complications (encephalopathy and convulsion) among 3506 term, singleton neonates with cord arterial $\mathrm{pH}<7.20$ (12). Neonatal death was much more likely at $\mathrm{pH}<7.00$. The cutoff value at which seizures became more likely was $\mathrm{pH}<7.05$, and for unexplained seizures $\mathrm{pH}<7.00$. They suggested that a realistic value for defining pathological acidemia was $\mathrm{pH}<7.00$. 
In Williams et al. study, neonatal seizure was predicted only by low umbilical artery $\mathrm{pH}$. A pH of less than 7 was more (73.8\%) sensitive than a base excess of -16 (52.5\%) in predicting the development of neonatal seizures (13). In our study only two cases of the acidemic neonates developed seizure both of whom had pH 6.99, 7.08 and base excess of -12.7 and -9.2 .

Victory et al. found that there is a progression of risk in term infants for NICU admission and need for assisted ventilation with worsening acidosis at birth (7).

Not all neonates with acidosis at birth require resuscitation or have adverse outcome. In the present study 60 percent did not require admission. King et al. concluded that infants with an umbilical artery $\mathrm{pH} \leq 7.0$ and assessed to be clinically well can be treated similar to nonacidemic infants (14). The cut off value for $\mathrm{pH}$ in our study is somewhat higher than in King's study.

In a prospective study performed by Loh and his colleagues on umbilical cord ABG analysis in labor ward on 200 neonates with purpose to establish the normal range of umbilical cord blood gas values, only 5 cases had umbilical cord $\mathrm{pH}$ under 7.05. An important point in this study was correlation between the significant metabolic acidosis compared to respiratory acidosis and poor outcome like prolonged stay in NICU and death (6). The poorest outcome in our study belonged to a baby who had received PPV at the time of birth and had pH less than 6.99 and base excess of -12.7.

Malin and her colleagues in their study (2010) declared that umbilical cord pH less than 7.2 was an appropriate prognostic agent for presenting asphyxia and other short outcomes. In their systematic review they showed that low arterial cord $\mathrm{pH}$ was significantly associated with perinatal and long term neonatal outcomes like mortality, hypoxic ischemic encephalopathy, intraventricular hemorrhage or periventricular leucomalacia and cerebral palsy (15).

Williams and Singh (2004) studied short term outcomes in 47 acidemic neonates. Early convulsion as most clear short outcome had been seen in moderate to severe encephalopathy in their study. Interestingly upper $\mathrm{PO}^{2}$ in their patients corresponded with poorer short outcomes, so they took $\mathrm{PO}^{2}$ for a poor prognostic agent. Our study didn't establish this opinion. In their study there was no correlation with $\mathrm{pH}, \mathrm{BD}, \mathrm{HIE}$ and convulsive disorder (16). These findings differ from those of ours and majority of researchers.

Bekedam and his colleagues in 2002 with the purpose of evaluating incidence of prenatal acidemia carried out a prospective cohort study. They showed that perinatal acidemia was more common in male neonates $(\mathrm{P}<0.0001)$ and male neonates were more prone to fetal distress and low Apgar score (17). This finding corresponds with our study, which showed mild predominance of perinatal acidemia incidence in male newborns.

One of limitations which may be attributed to our study may be related to study population, all of whom were mother-baby pairs from cesarean section group. The umbilical cord $\mathrm{pH}$ value may differ between cesarean section and normal vaginal delivery groups as indicated by Loh et al. In their study, the mean and standard deviation values of umbilical artery $\mathrm{pH}$ were 7.21 and 0.08 for vaginal deliveries, and 7.22 and 0.07 for caesarean sections, respectively (6).

The results of present study showed that short term outcomes like need for advanced resuscitation, NICU admission, HIE, delay to start oral feeding, mean hospital stay were higher in acidemic in comparison to non-acidemic neonates. Therefore, an umbilical cord PH less than 7.2 immediately after birth can be used as a prognostic factor for prediction of unfavorable short term outcomes in newborns.

\section{Acknowledgements}

We would like to thank Dr Firozjahi for his laboratory support.

\section{Funding/Support}

This study was funded by Research deputy of Babol University of Medical Sciences (Proposal No: 1294).

\section{References}

1. White CR, Doherty DA, Henderson JJ, Kohan R, Newnham JP, Pennell CE. Benefits of introducing universal umbilical cord blood gas and lactate analysis into an obstetric unit. Aust N Z J Obstet Gynaecol. 2010;50(4):318-28.

2. Vukojevic M, Soldo I, Granic D. Risk factors associated with cerebral palsy in newborns. Coll Antropol. 2009;33 Suppl 2:199-201.

3. Royal College of Obstetricians and Gynaecologists. The use of electronic fetal monitoring: the use and interpretation of cardiotocography in intrapartum fetal surveillance. In: National Institute for Health and Care Excellence, editor. London: 2001.

4. Acog Committee on Obstetric Practice. ACOG Committee Opinion No. 348, November 2006: Umbilical cord blood gas and acidbase analysis. Obstet Gynecol. 2006;108(5):1319-22.

5. Armstrong L, Stenson BJ. Use of umbilical cord blood gas analysis in the assessment of the newborn. Arch Dis Child Fetal Neonatal Ed.2007;92(6):F430-4.

6. Loh SF, Woodworth A, Yeo GS. Umbilical cord blood gas analysis at delivery. Singapore Med J.1998;39(4):151-5.

7. Victory R, Penava D, Da Silva O, Natale R, Richardson B. Umbilical cord $\mathrm{pH}$ and base excess values in relation to adverse outcome events for infants delivering at term. Am J Obstet Gynecol. 2004;191(6):2021-8.

8. Ip FF, di Pierro I, Brown R, Cunningham I, Handelsman DJ, Liu PY. Trough serum testosterone predicts the development of polycythemia in hypogonadal men treated for up to 21 years with subcutaneous testosterone pellets. Eur J Endocrinol. 2010;162(2):385-90.

9. Kattwinkel J. NRP Neonatal Resuscitation Textbook 6th Edition (English version). 6th Edition ed:American Academy of Pediatrics and American Heart Association Guidelines for Neonatal Resuscitation; 2011.

10. Levene MI, DeVries LS. Hypoxic-ischemic encephalopathy. In: Martin RJ, Fanaroff AA, Walsh MC editors. Fanaroff and Martin's Neonatal-Perinatal Medicine: Diseases of the Fetus and Infant.: Elsevier Health Sciences; 2010.

11. Andres RL, Saade G, Gilstrap LC, Wilkins I, Witlin A, Zlatnik F, et al. Association between umbilical blood gas parameters and neonatal morbidity and death in neonates with pathologic fetal acidemia. Am J Obstet Gynecol. 1999;181(4):867-71.

12. Goldaber KG, Gilstrap L3, Leveno KJ, Dax JS, McIntire DD. Patho- 


\section{Ahmadpour-Kacho M et al.}

logic fetal acidemia. Obstet Gynecol.1991;78(6):1103-7.

13. Williams KP, Singh A. The correlation of seizures in newborn in fants with significant acidosis at birth with umbilical artery cord gas values. Obstet Gynecol. 2002;100(3):557-60.

14. King TA, Jackson GL, Josey AS, Vedro DA, Hawkins H, Burton $\mathrm{KM}$, et al. The effect of profound umbilical artery acidemia in term neonates admitted to a newborn nursery. $J$ Pediatr. 1998;132(4):624-9.

15. Malin GL, Morris RK, Khan KS. Strength of association between umbilical cord $\mathrm{pH}$ and perinatal and long term outcomes: systematic review and meta-analysis. BMJ.2010;340:c1471.

16. Williams KP, Singh A. Umbilical artery blood gas parameters in neonates with early onset seizures who die. BJOG. 2004;111(10):1042-5.

17. Bekedam DJ, Engelsbel S, Mol BW, Buitendijk SE, van der Pal-de Bruin KM. Male predominance in fetal distress during labor. Am J Obstet Gynecol. 2002;187(6):1605-7. 